\title{
PHGDH as a mechanism for resistance in metabolically-driven cancers
}

\author{
Richa Rathore ${ }^{1}$, Charles R. Schutt ${ }^{1}$, Brian A. Van Tine ${ }^{1,2}$ \\ 'Division of Medical Oncology, Washington University in St. Louis, St. Louis, MO 63110, USA. \\ ${ }^{2}$ Siteman Cancer Center, St. Louis, MO 63110, USA.
}

Correspondence to: Dr. Brian A. Van Tine, Associate Professor of Medicine, Division of Medical Oncology, Washington University in St. Louis, 660 S. Euclid Ave., Campus Box 8007, St. Louis, MO 63110, USA. E-mail: bvantine@wustl.edu

How to cite this article: Rathore R, Schutt CR, Van Tine BA. PHGDH as a mechanism for resistance in metabolically-driven cancers. Cancer Drug Resist2020;3:762-74. http://dx.doi.org/10.20517/cdr.2020.46

Received: 28 Jun 2020 First Decision: 16 Jul 2020 Revised: 28 Jul 2020 Accepted: 21 Aug 2020 Available online: 17 Sep 2020

Academic Editor: Ramandeep Rattan Copy Editor: Cai-Hong Wang Production Editor: Jing Yu

\begin{abstract}
At the forefront of cancer research is the rapidly evolving understanding of metabolic reprogramming within cancer cells. The expeditious adaptation to metabolic inhibition allows cells to evolve and acquire resistance to targeted treatments, which makes therapeutic exploitation complex but achievable. 3-phosphoglycerate dehydrogenase (PHGDH) is the rate-limiting enzyme of de novo serine biosynthesis and is highly expressed in a variety of cancers, including breast cancer, melanoma, and Ewing's sarcoma. This review will investigate the role of PHGDH in normal biological processes, leading to the role of PHGDH in the progression of cancer. With an understanding of the molecular mechanisms by which PHGDH expression advances cancer growth, we will highlight the known mechanisms of resistance to cancer therapeutics facilitated by PHGDH biology and identify avenues for combatting PHGDH-driven resistance with inhibitors of PHGDH to allow for the development of effective metabolic therapies.
\end{abstract}

Keywords: PHGDH, cancer, metabolism, serine, one-carbon metabolism, folate cycle, drug resistance

\section{INTRODUCTION}

Recent advances in anti-cancer treatments have been based on the increased identification of biomarkers that allow for tumour-specific therapy. Biomarker-driven therapies allow for the differentiation between cancer and host cells, with the potential to decrease the side-effects often associated with chemotherapy

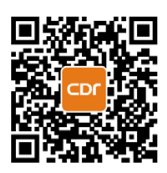


in normal tissue. The hallmarks of cancer, such as rapid growth, sustained proliferation, and increased invasion and metastasis, can be traced to the activation or suppression of oncogenes, which can then be used as biomarkers for targeting therapeutics ${ }^{[1]}$.

The progression of cancer is dependent on the cellular metabolism of the tumour ${ }^{[2]}$. As such, developing therapeutic methods that target tumour metabolism has been a growing field. One-carbon metabolism is of importance in cancer metabolism, as this pathway is necessary for the de novo generation of biomass and other nutrient precursors. One-carbon metabolism consists of serine biosynthesis, betaine biosynthesis, the folate cycle, and the methionine cycle. The products of one-carbon metabolism contribute to nucleotide, lipid, and methylation metabolism, as well as nicotinamide adenine dinucleotide phosphate (NADPH), reactive oxygen species (ROS), and glutathione synthesis ${ }^{[3,4]}$. Of these, the serine biosynthetic pathway is of interest because the rate limiting enzyme, 3-phosphoglycerate dehydrogenase (PHGDH), is highly expressed in a variety of cancers and contributes to drug resistance. Both of these facets of PHGDH metabolism are discussed in detail below.

Importantly, tumour metabolism is highly adaptable, and the metabolic systems of cancer cells can reprogram in response to nutrient and anabolic precursor availability. As a result, there is a risk of innate or acquired drug resistance to metabolic inhibitors. In this review, the requirement of serine synthesis for cancer metabolism and tumour progression is explored. Through the biology of PHGDH, mechanisms of resistance to current cancer treatments, as well as proposed novel treatments, are identified. Understanding the mechanisms of resistance to metabolic treatments allows for the design of conditionally lethal combination therapies based on the inherent properties of tumour metabolism that can combat acquired resistance.

\section{THE BIOLOGICAL ROLE OF PHGDH IN CANCER}

\section{PHGDH in the untransformed cell}

PHGDH is the rate-limiting enzyme of de novo serine biosynthesis. PHGDH catalyses the conversion of the glycolytic intermediate 3-phosphoglycerate (3PG) to 3-phosphohydroxypyruvate (3PHP). The PHGDH enzymatic reaction utilizes nicotinamide adenine dinucleotide (oxidized form, $\mathrm{NAD}^{+}$; reduced form, $\mathrm{NADH}$ ) as a cofactor, generating $\mathrm{NADH}$ as $3 \mathrm{PHP}$ is biosynthesized. Phosphoserine aminotransferase (PSAT1) subsequently uses glutamate to confer a nitrogen unit onto $3 \mathrm{PHP}$, producing alpha-ketoglutarate $(\alpha K G)$ in addition to the serine precursor, 3-phosphoserine (3PS $)^{[5]}$. Finally, phosphoserine phosphatase converts 3 PS to serine [Figure 1] $]^{[6]}$.

Serine is required for a variety of biosynthetic and signalling processes [Figure 2]. Serine itself can be used for protein and lipid biosynthesis ${ }^{[7]}$. The removal of a methylene unit from serine by serine hydroxymethyltransferase (cytosolic, SHMT1; mitochondrial, SHMT2) results in the synthesis of other amino acids, including glycine and, through intermediates of the methionine cycle, cysteine $e^{[8,9]}$. The methylene unit from serine also serves as a one-carbon donor for the folate cycle. The products of the folate cycle and the methionine cycle contribute to purine and pyrimidine synthesis, homocysteine recycling for DNA methylation processes, and the generation of NADH, NADPH, and adenosine triphosphate (ATP) ${ }^{[10,11]}$. Additionally, de novo serine biosynthesis utilizes glutamate and produces $\alpha \mathrm{KG}$, which can be converted to D-2-hydroxyglutarate (D-2HG), an oncometabolite ${ }^{[5]}$. As a result, the increased expression of PHGDH, as well as the other enzymes in the serine biosynthetic pathway, indicates that cells are utilizing these processes for proliferation and production of biomass.

Serine also plays a role in downstream signalling in the cell. When activated, pyruvate kinase (PK) catalyses the conversion of phosphoenolpyruvate to pyruvate, and is a key checkpoint for glycolysis ${ }^{[12]}$. As such, PK serves an important role in the occurrence of the Warburg effect, that describes tumour 


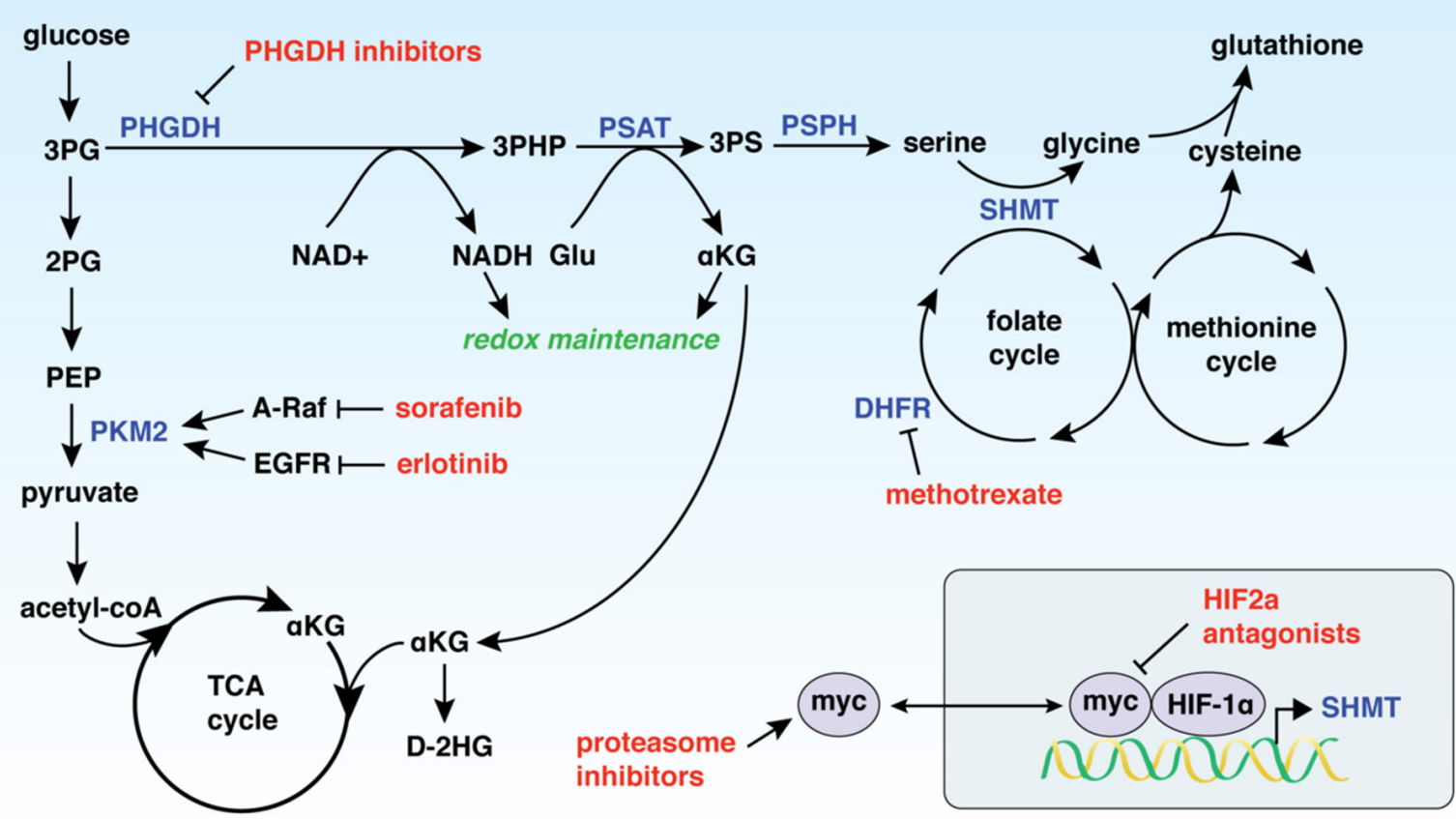

Figure 1. PHGDH expression drives resistance to a variety of chemotherapeutics (shown in red) through modulation of metabolic pathways. 3PG: 3-phosphoglycerate; 2PG: 2-phosphoglycerate; PEP: phosphoenolpyruvate; PKM2: pyruvate kinase M2; TCA cycle: citric acid cycle; $\alpha$ KG: $\alpha$-ketoglutarate; D-2HG: D-2-hydroxyglutarate; PHGDH: 3-phosphoglycerate dehydrogenase; 3-PHP: 3-phosphohydroxypyruvate; PSAT: phosphoserine aminotransferase; 3PS: 3-phosphoserine; PSPH: phosphoserine phosphatase; $N A D^{+}$: nicotinamide adenine dinucleotide, oxidized; NADH: nicotinamide adenine dinucleotide, reduced; Glu: glutamate; SHMT: serine hydroxymethyltransferase; DHFR: dihydrofolate reductase

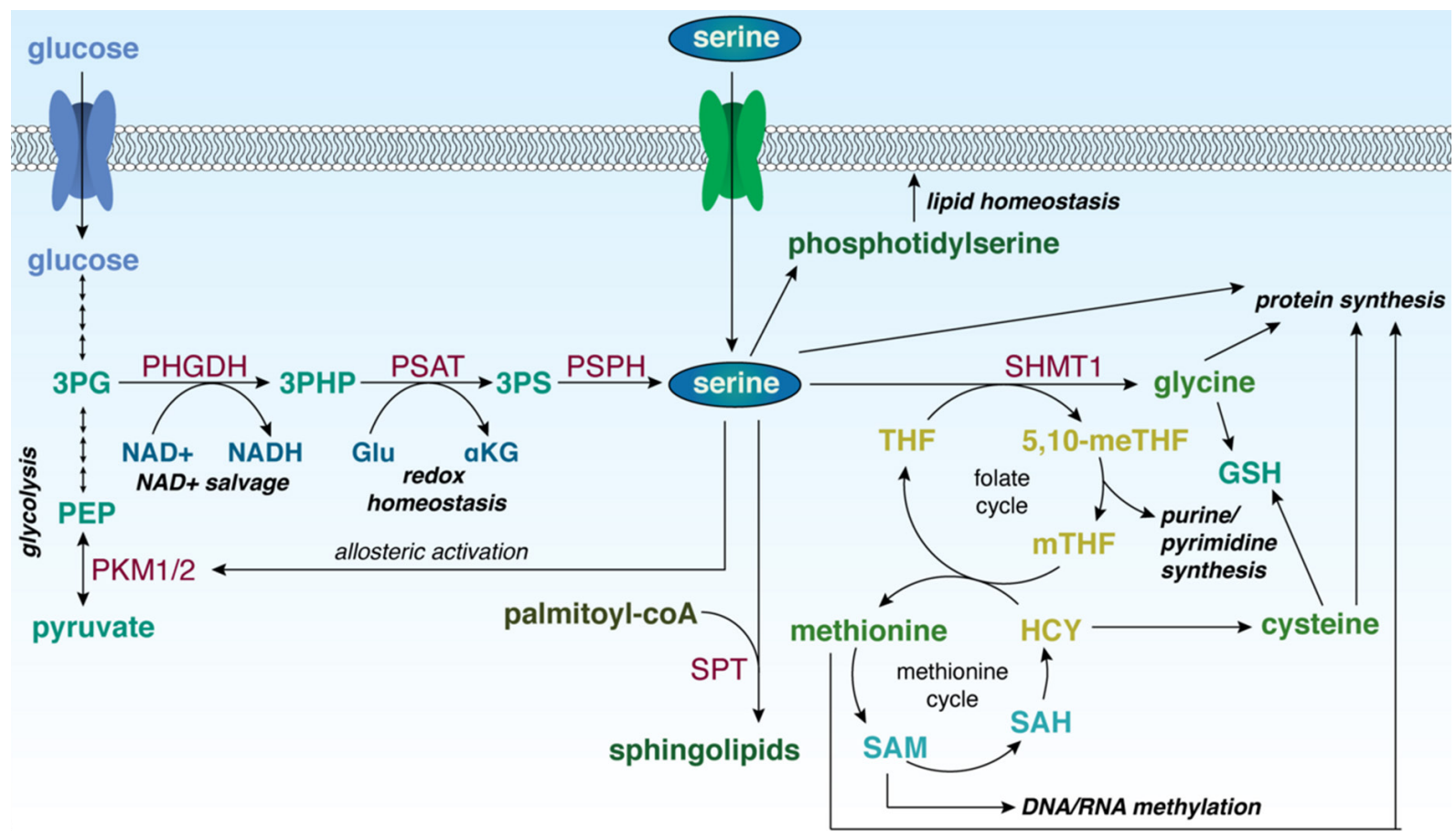

Figure 2. Role of serine in biological processes in the cell. 3PG: 3-phosphoglycerate; PEP: phosphoenolpyruvate; PKM1/2: pyruvate kinase isoforms M1/M2; PHGDH: 3-phosphoglycerate dehydrogenase; 3-PHP: 3-phosphohydroxypyruvate; PSAT: phosphoserine aminotransferase; 3PS: 3-phosphoserine; PSPH: phosphoserine phosphatase; $\mathrm{NAD}^{+}$: nicotinamide adenine dinucleotide, oxidized; NADH: nicotinamide adenine dinucleotide, reduced; Glu: glutamate; $\alpha$ KG: $\alpha$-ketoglutarate; SPT: serine palmitoyltransferase; SHMT1: serine hydroxymethyltransferase 1; 5,10-meTHF: 5,10-methylene tetrahydrofolate; MTHF: 5-methyl tetrahydrofolate; THF: tetrahydrofolate; GSH: reduced glutathione; HCY: homocysteine; SAH: S-adenosyl homocysteine; SAM: S-adenosyl methionine 
cells as more highly glycolytic than oxidative ${ }^{[13]}$. In its inactive form, PK shunts glucose carbons back through glycolytic intermediates, supporting the utilization of glucose for biomass synthesis rather than mitochondrial energy production ${ }^{[14]}$. PK exists in the body in two isoforms (isoform M1, PKM1; isoform M2, PKM2). PKM1 is mainly found in skeletal muscle and brain cells. PKM2 is expressed at a significantly higher ratio in proliferating cells and is the more dominant isoform in cancer; however, PKM1 has been shown to be expressed in certain cancers and cancer-associated fibroblasts as well ${ }^{[13,15]}$. Importantly, PKM2 is enzymatically activated by the direct binding of serine to an allosteric site ${ }^{[16]}$. Inactive PKM2 can therefore respond to changes in serine availability and direct glycolytic intermediates through $3 \mathrm{PG}$ into the biosynthesis of serine, activating PKM2. PKM2 expression is also mediated by a series of pathways, including phosphatidylinositol-3-kinase (PI3K)/AKT/mammalian target of rapamycin (mTOR) ${ }^{[14,17]}$.

\section{Incidences of decreased PHGDH}

Serine deficiency can be extremely detrimental to cells, resulting in limited cellular proliferation and cell cycle arrest ${ }^{[18]}$. The first report of PHGDH deficiency was described in 1996, where two brothers with decreased plasma concentrations of serine and glycine presented with a severe neurological syndrome ${ }^{[19]}$. This decrease in serine and glycine was associated with decreased PHGDH expression and activity in the brains of the patients. Importantly, subsequent studies found that, although alternate pathways of obtaining serine exist, PHGDH deficiency resulted in significantly lower plasma serine level ${ }^{[20]}$. Later studies found that phenotypes of PHGDH deficiency can exist on a spectrum and identified Neu-Laxova syndrome as a more severe example of PHGDH deficiency. Neu-Laxova syndrome is an autosomal recessive disorder caused by mutations to PHGDH and subsequent loss of serine, and is characterized by neurological impairment, impaired fetal development, and skeletal anomalies ${ }^{[20,21]}$.

At the cellular level, PHGDH deficiency can result in loss of DNA methylation. As the methylene unit provided to the folate cycle by the conversion of serine to glycine can transfer into the methionine cycle, serine indirectly supports the recycling of homocysteine to methionine, as well as the generation of precursors for S-adenosylmethionine $(\mathrm{SAM})^{[22,23]}$. SAM is a common methyl-group donor required for DNA methylation ${ }^{[24]}$. In acute serine-starvation conditions, SAM is no longer used to methylate DNA and $\mathrm{RNA}^{[25]}$. Importantly, metabolic remodelling in cells during acute serine starvation allow for the upregulation of serine biosynthesis and serine uptake, to compensate for this $\operatorname{loss}^{[26]}$.

De novo serine biosynthesis also provides the precursors for the generation of phosphatidylserine and sphingolipids; PHGDH deficiency can alter sphingolipid homeostasis by prompting the generation of deoxysphingolipids ${ }^{[27,28]}$. Sphingolipids are produced by the enzyme serine palmitoyltransferase (SPT) that incorporates serine into palmitoyl-coA to produce a precursor to sphingosine. With decreased environmental serine, SPT instead utilizes alanine as a cofactor, resulting in the generation of deoxysphingolipids ${ }^{[27,29]}$. Deoxysphingolipids cannot be incorporated into cellular membranes, and increased levels of deoxysphingolipids can result in mitochondrial dysfunction ${ }^{[30]}$.

The results of decreased serine and loss of PHGDH activity are generally not beneficial to cells, unless extracellular serine concentrations are sufficiently high to support cellular utilization of the amino acid. The mechanism of negative regulation of PHGDH, therefore, is critical to explore. In melanoma, $P H G D H$ expression is transcriptionally downregulated by wildtype $\mathrm{p}_{53} 3^{[31]}$. It was found that $P H G D H$ was a transcriptional target of the tumour suppressor p53, and that suppression of $P H G D H$ resulted in promotion of apoptosis in p53-wildtype melanoma. These findings indicate the importance of PHGDH in the baseline functioning of the cell, but also highlight the utility of increased PHGDH as an oncogene in cancer.

\section{THE TUMORIGENIC CONSEQUENCES OF ELEVATED PHGDH}

The increased expression of serine synthetic enzymes can signal that a cell is proliferating and generating biomass at a rapid rate, a hallmark of cancer ${ }^{[32]}$. PHGDH has been demonstrated to be upregulated in a wide 
variety of biologically distinct cancers, including colorectal cancer ${ }^{[33]}$, gastric cancer ${ }^{[34]}$, breast cancer ${ }^{[35,36]}$, melanoma $^{[37]}$, Ewing's sarcoma ${ }^{[38]}$, cervical cancer ${ }^{[39]}$, pancreatic cancer ${ }^{[40]}$, thyroid cancer ${ }^{[41]}$, colon cancer ${ }^{[42]}$, lung adenocarcinoma ${ }^{[43]}$, and non-small cell lung cancer ${ }^{[44]}$. Furthermore, increased PHGDH expression has been linked to brain metastasis ${ }^{[45]}$.

\section{Mutations to TP53}

As previously stated, wildtype p53 can transcriptionally decrease gene expression of $P H G D H^{[31]}$. Most of the cancers that present an overexpression of PHGDH harbour TP53 mutations, including colorectal cancer (55\%-60\% TP53 mutation), intestinal gastric cancer (66\% TP53 mutation), melanoma (85\% TP53 mutation), and non-small cell lung cancer $(50 \% \text { TP53 mutation })^{[46-49]}$. This suggests that cancers with non-wildtype TP53 may have increased PHGDH expression, though this has not yet been explored. Additionally, mutant p53 can regulate PKM2 through an mTOR-mediated phosphorylation at Tyr $105^{[50,51]}$. The effects that mutant p53 can have on serine synthetic enzymes and downstream metabolic enzymes can contribute to mutant p53-driven tumorigenesis.

\section{Cell growth and proliferation}

Increased PHGDH activity results in increased de novo serine biosynthesis. As previously described, serine supports a variety of cellular processes, including amino acid, nucleotide, and lipid synthesis, increased DNA methylation, and indirect $\alpha$ KG generation. De novo serine biosynthesis can in turn drive the synthesis of glycine, as well as the synthesis of cysteine from homocysteine within the methionine cycle, supporting protein synthesis ${ }^{[8,52]}$. Glycine is also directly incorporated into purine nucleotides. Furthermore, serine can be incorporated into lipids to produce phosphoserine and is a precursor to sphingosine, from which all sphingolipids are derived ${ }^{[53]}$. These processes support the generation of biomass and nucleic acid replication for the rapid proliferation of cancer cells.

\section{Redox homeostasis}

The increased production of glycine and cysteine from serine can also contribute to maintenance of redox balance in cells, as these are the precursors for glutathione ${ }^{[54]}$. Glutathione, which exists in a reduced form (GSH) and an oxidized form (GSSG is the primary ROS scavenger of the cell ${ }^{[55]}$. Generation of $\alpha \mathrm{KG}$, an essential component of the citric acid (TCA) cycle, also contributes to maintaining redox balance, as $\alpha \mathrm{KG}$ has antioxidative functions in the cell ${ }^{[56]}$. Furthermore, PHGDH can directly catalyse the conversion of $\alpha \mathrm{KG}$ to D-2HG, an oncometabolite ${ }^{[5]}$. This reverse enzymatic activity requires the oxidation of NADH to $\mathrm{NAD}^{+}$, an important co-factor in metabolism and redox homeostasis ${ }^{[5,57]}$.

\section{The NAD ${ }^{+}$salvage pathway}

PHGDH utilizes $\mathrm{NAD}^{+}$as a co-factor for enzymatic activity, producing NADH during the synthesis of $3 \mathrm{PHP}$ from $3 \mathrm{PG}^{[58]}$. In order to be utilized, $\mathrm{NAD}^{+}$must be continually synthesized from tryptophan or regenerated from $\mathrm{NADH}$. The $\mathrm{NAD}^{+}$salvage pathway occurs through the recycling of nicotinamide to nicotinamide mononucleotide, and is therefore required for functional serine biosynthesis ${ }^{[59]}$. Conversely, mitochondrial serine catabolism has been demonstrated to supplement NADH levels through the folate cycle, suggesting that PHGDH and serine metabolism are directly regulated by $\mathrm{NAD}^{+} / \mathrm{NADH}$ availability ${ }^{[6]}$.

\section{Metastasis}

Increased PHGDH expression has been demonstrated to not only promote cancer growth and proliferation, but also drive secondary tumour formation and metastasis ${ }^{[6]]}$. In a study on lung metastasis, increased PHGDH increased hypoxia-inducible factor (HIF)-target gene expression. As increased PHGDH results in elevated production of glutathione, the resultant hypoxic conditions could be maintained by glutathione, subsequently executing metastatic programs ${ }^{[61]}$. Furthermore, in a study in brain metastases, increased PHGDH expression was correlated with increased metastatic potential to the brain. Interestingly, in this 
study, inhibiting PHGDH attenuated metastasis without affecting extra-cranial tumour growth, suggesting that the consequences of increased PHGDH expression were directly related to upregulated metastasis ${ }^{[45]}$. Finally, this study highlighted the limited environmental availability of serine in the brain, demonstrating the subsequent reliance on de novo serine biosynthesis ${ }^{[45]}$.

Taken together, baseline PHGDH expression contributes to de novo serine biosynthesis in the cell and supports a multitude of cellular pathways. Overexpression of PHGDH drives numerous pathways that are particularly useful for the initiation and progression of cancer.

\section{THE ROLE OF INCREASED PHGDH IN CANCER DRUG RESISTANCE}

Given that increased PHGDH contributes to tumorigenesis, the role of PHGDH in cancer resistance is multi-faceted. Elevated PHGDH expression drives a reliance on certain metabolic pathways that cancer therapeutics directly target, thus resulting in a series of inhibitors to which cancers with increased PHGDH can develop resistance [Figure 1].

\section{Tyrosine kinases}

Tyrosine kinases catalyse the phosphorylation of tyrosine residues, and have been shown to be constitutively active in oncogenic program ${ }^{[62]}$. Sorafenib targets multiple tyrosine kinases, primarily the rapidly accelerated fibrosarcoma kinase (RAF) pathway, but also the vascular endothelial growth factor receptor and platelet-derived growth factor receptor pathways ${ }^{[63,64]}$. The inhibition of RAF-1 by sorafenib leads to inhibition of cellular proliferation and tumour growth. RAF-1 inhibition elevates ROS levels through stimulation of the Raf/MEK/Erk pathway, causing apoptosis ${ }^{[65]}$. Sorafenib has been approved for use in hepatocellular carcinoma (HCC), renal cell cancer, and thyroid cancer ${ }^{[66]}$. A study exploring the mechanisms driving sorafenib resistance found that increased PHGDH expression was a critical for this process in $\mathrm{HCC}^{[64]}$. As $\mathrm{PHGDH}$ and the serine synthesis pathway generate antioxidants (including glutathione and $\alpha \mathrm{KG}$ ), elevated PHGDH can combat the increased ROS levels induced by sorafenib treatment, thereby repressing apoptosis. Additionally, A-RAF, a RAF paralog, increases activation of PKM2 in the presence of serine, offering an additional target for sorafenib and another resistance mechanism in PHGDH-overexpressed cancers ${ }^{[14,67]}$.

\section{Epidermal growth factor receptor}

Erlotinib is an inhibitor of epidermal growth factor receptor (EGFR), another tyrosine kinase associated with a number of signalling cascade pathways, including the Ras/Raf/MEF/ERK, PI3K/Akt, and STAT pathways ${ }^{[68]}$. The MEF/ERK pathway in particular links EGFR signalling to increased glycolysis, and Akt pathway signalling links EGFR to increased PKM2 activity ${ }^{[69,70]}$. EGFR has been demonstrated to be a driver of lung adenocarcinoma, and erlotinib treatment has been approved for treatment of non-small cell lung cancer in patients with and without EGFR mutations ${ }^{[71,72]}$.

Erlotinib binds to EGFR and inhibits downstream signalling cascades. Disruption of these signalling cascades results in decreased cell cycle progression, oxidative stress, and apoptosis. PHGDH is upregulated in erlotinib-resistant lung adenocarcinomas, likely due to the upregulation of glutathione and $\alpha \mathrm{KG}$ synthesis as a cellular response to oxidative stress ${ }^{[73]}$. Furthermore, increased PHGDH expression and serine biosynthesis drives increased PKM2 activity and glycolysis, increasing the utilization of EGFRrelated pathways.

\section{HIFs}

HIFs contain two subunits that have transcription factor activity in hypoxic cells. The $\alpha$-subunit HIF $2 \alpha$ mediates redox homeostasis and can therefore modulate the effects of drugs such as sorafenib by decreasing resultant ROS levels and improving oxygen supply ${ }^{[74]}$. HIF2 $\alpha$ is regulated by $\mathrm{c}-\mathrm{Myc}$ activation and promotes 
hypoxic cell proliferation ${ }^{[75]}$. Therapies targeting HIF $2 \alpha$ have therefore been explored as replacements for some tyrosine kinase inhibitors, such as sunitinib, in clear-cell renal cell carcinoma ${ }^{[76]}$. PHGDH is significantly overexpressed in HIF2 $\alpha$ knockout tumours, as well as tumours that have shown sunitinib resistance $^{[76]}$. The redox homeostasis maintenance conferred by PHGDH overexpression may be implicated here.

Interestingly, HIF $1 \alpha$, but not HIF2 $\alpha$, can also regulate the expression of SHMT, the enzyme that converts serine to glycine ${ }^{[77]}$. Increased SHMT1/2 expression can drive serine catabolism, increasing mitochondrial $\mathrm{NADH}$ production and fuelling the $\mathrm{NAD}^{+}$salvage pathway required for PHGDH activity and serine biosynthesis $^{[59,77]}$.

\section{The proteasome}

Based on the potential role of c-Myc in resistance to HIF2 $\alpha$ inhibitors, the role of NMYC in systems associated with PHGDH has been explored. NMYC activates ATF4, subsequently increasing PHGDH expression and activating a dependence on the serine biosynthetic pathway ${ }^{[78-80]}$. Proteasome inhibitors downregulate c-Myc, and have therefore been utilized to combat c-Myc-driven cancers ${ }^{[81]}$. Bortezomib is a proteasome inhibitor that has been highly effective for the treatment of multiple myeloma, a cancer in which c-Myc is highly active ${ }^{[82]}$. Proteasome inhibition by bortezomib results in the accumulation of unfolded proteins in the endoplasmic reticulum, resulting in cell death from the overproduction of reactive oxygen species $(\mathrm{ROS})^{[83]}$. PHGDH is upregulated in bortezomib-resistant multiple myeloma; interestingly, this mechanism has also been identified as being through increased glutathione synthesis ${ }^{[84-86]}$ and subsequent ROS scavenging.

\section{Mitogen-activated protein kinase kinase}

Mitogen-activated protein kinases (MAPK) and extracellular signal-regulated kinases (ERK) make up a series of proteins that transduce signals from the extracellular environment to inform cellular processes. This pathway can be overactive in some cancers, and activates transcription factors that are responsible for the progression of cancers such as melanoma ${ }^{[87,88]}$. In melanoma, tumour growth is enhanced through activation of the MAPK pathway, which is primarily driven by activating mutations in two oncogenes: $B R A F$ and NRAS ${ }^{[89]}$. Mitogen-activated protein kinase kinase (MEK) enzymes are a part of the MAPK/ERK pathway, and activate the final kinases in this signalling pathway ${ }^{[88]}$. MEK inhibitors target this pathway and inhibit cell proliferation, ultimately causing apoptosis ${ }^{[90]}$. Importantly, BRAF mutations can increase susceptibility to MEK inhibitors; however, NRAS mutations that can over-activate MEK/ERK signalling can lead to resistance to MEK inhibitors ${ }^{[87,91]}$.

In an NRAS mutation model of melanoma, PHGDH was found to be upregulated. PHGDH is upregulated in melanoma at baseline, and is also upregulated in MEK inhibitor-resistant melanomas ${ }^{[91,92]}$. Overactive MEK/ERK signalling in BRAF- and NRAS-mutated cancers can overexpress PHGDH through ATF4 activation, driving utilization of the serine synthesis pathway to generate glutathione as a resistance mechanism. In addition, increased levels of folic acid, which can occur through increased flux of carbons from serine synthesis through the folate cycle, is a possible mechanism of resistance to MEK inhibitors in BRAF inhibitor-resistant melanomas ${ }^{[91,92]}$.

\section{Cisplatin}

PHGDH is elevated in cervical adenocarcinoma, and is associated with poorer prognosis ${ }^{[93]}$. The firstline therapy for cervical adenocarcinoma is platinum-based chemotherapy. Cisplatin induces the DNA damage response in cells and causes mitochondrial apoptosis through $\mathrm{Bcl} 2{ }^{[93]}$. Given that PHGDH drives the indirect synthesis of nucleotides, upregulation of PHGDH could improve the DNA damage response in cells treated with cisplatin. PHGDH knockdown in cervical adenocarcinoma resulted in a decrease in Bcl2 
expression, suggesting that baseline high PHGDH could also result in increased $\mathrm{Bcl}$, thereby mitigating the mitochondrial apoptotic response ${ }^{[93]}$. Interestingly, in ovarian carcinoma, increased PHGDH is a marker of cisplatin sensitivity, rather than resistance ${ }^{[94]}$. Further exploration of this and other pathways involved in cisplatin resistance is therefore needed.

\section{Other therapies}

Elevated PHGDH also has the potential to play a role in resistance to therapies that have not yet been mechanistically explored. A critical therapy to therefore mention is the use of folate cycle inhibitors such as methotrexate and raltitrexed ${ }^{[95]}$. Methotrexate targets dihydrofolate reductase (DHFR), while raltitrexed targets thymidylate synthase (TYMS). These drugs require functioning folate cycles in order to be effective, and increased expression of folate-related enzymes is highlighted as a current mechanism of resistance ${ }^{[96,97]}$. As increased serine synthetic pathway activity can contribute more methyl units to the folate cycle, elevated PHGDH could be directly related to resistance to methotrexate treatment.

\section{THE INHIBITION OF PHGDH TO COMBAT CHEMO-RESISTANCE}

\section{Single-agent inhibition of PHGDH}

Given the increased expression of PHGDH in a variety of cancers, the single-agent inhibition of PHGDH seems to be a promising prospect for cancer therapy. A series of small molecule inhibitors against PHGDH have been developed, primarily targeting the enzymatic activity of PHGDH. These inhibitors include NCT-503, CBR-5884, PKUMDL-WQ-2101, BI-4924, and others under preclinical and clinical development ${ }^{[35,98-102]}$. It is important for the field to validate any finding with small-molecule inhibitors with knockdown and rescue or structural analysis to ensure that the effects of the drug are as a result of PHGDH biology and not an off-target effect of small-molecule inhibitors as a class of inhibitors. Iterations of these compounds have shown increasingly less off-target effects, with structure-based approaches used to synthesize PKUMDL-WQ-2101 in order to confirm specific binding to PHGDH ${ }^{[101]}$. PKUMDL-WQ-2101 and NCT-503 have been widely used in in vitro and in vivo research to interrogate the role of PHGDH and serine metabolism in normal and cancer cells ${ }^{[101,103]}$.

When cancer cells with elevated PHGDH expression are treated with high doses of NCT-503, cellular proliferation is attenuated and, in some cases, cell death is observed ${ }^{[38,85,103]}$. This phenotype can also be observed in vivo, as numerous studies have demonstrated that NCT-503 treatment results in decreased tumour growth for tumours and cell line-derived xenografts of PHGDH-high cancers. Knockout studies of PHGDH have also suggested mild suppressive effects on proliferation ${ }^{[64]}$. Furthermore, metabolites downstream of the serine biosynthetic pathway, such as one carbon units, folate intermediates, and pyrimidine intermediates, were also dysregulated by PHGDH inhibition ${ }^{[102,103]}$.

As of yet, direct PHGDH inhibition has not been tested in human clinical studies. However, as cells with increased PHGDH expression can develop resistance to apoptotic cell death by various drug treatments, cells treated with PHGDH inhibitors can rapidly alter their metabolism to take advantage of other mechanisms of fuel oxidation and redox maintenance ${ }^{[104]}$. As a result, PHGDH inhibition must be approached in a different way to optimize it for clinical development.

\section{DUAL-AGENT INHIBITION OF PHGDH AND ASSOCIATED PATHWAYS}

Given the highly adaptable nature of cancer metabolism, synergistic drug combinations are the future of metabolism-based drug resistance. Identifying increased PHGDH expression as a resistance mechanism for a variety of cancer therapeutics offers the opportunity to combine PHGDH inhibition with small molecule therapeutics. For example, increased PHGDH expression has been associated with both erlotinib and cisplatin resistance. Treatment with NCT-503 in these systems conferred sensitivity to the targeted therapy 
erlotinib and the chemotherapy cisplatin, respectively ${ }^{[73,93]}$. Furthermore, increased PHGDH expression was associated with resistance to sorafenib ${ }^{[64]}$. Treatment with NCT-503 mildly suppressed proliferation in hepatocellular carcinoma cells, but combining NCT-503 with sorafenib caused complete attenuation of proliferation and induced significant apoptosis ${ }^{[64]}$.

Beyond the known therapies that PHGDH confers resistance to, the combination of NCT-503 with the targeting of other cellular pathways can mitigate resistance. An understanding of the downstream mechanistic actions of PHGDH activity can unveil new therapies that could have action in PHGDHoverexpressed cancers. Given that PHGDH activity requires the $\mathrm{NAD}^{+}$salvage pathway, a study that explored the use of NCT-503 with a nicotinamide phosphoribosyltransferase (NAMPT) inhibitor. This combination with a NAMPT that blocks the $\mathrm{NAD}^{+}$salvage pathway resulted in synergistic cell death ${ }^{[38]}$. Recent studies have also explored the metabolic implications and pro-survival adaptations that occur as a result of PHGDH inhibition, implicating decreased TCA cycle activity, mTOR-independent and -dependent autophagy, and enhanced lipid metabolism and formation of lipid bodies ${ }^{[2,103,105,106]}$. The combination of PHGDH inhibition with inhibitors of these pro-survival metabolic adaptations should therefore yield synergistic and dramatic results in PHGDH-high cancers.

\section{CONCLUDING REMARKS}

3-phosphoglycerate dehydrogenase (PHGDH) expression in cancer has been linked to shorter progressionfree survival, increased rates of metastasis, and poorer overall survival. An in-depth analysis of the biological consequences of enhanced PHGDH expression shows the links between de novo serine biosynthesis and a series of metabolic pathways that are targeted by current chemotherapies. In particular, cancers are capable of developing resistance to chemotherapies that induce apoptosis through increased ROS by increasing PHGDH, as PHGDH generates the necessary metabolic precursors for antioxidant and ROS scavenging activity. Therefore, increased levels of PHGDH, while contributing to tumorigenicity, can contribute to the innate or acquired resistance of cancers to current chemotherapies.

The direct inhibition of PHGDH by small-molecule inhibitors results in a decrease in cellular proliferation in vitro, with marginal inhibition of tumour growth in vivo. Inhibition of PHGDH also results in a series of metabolic adaptations that can acutely sensitize tumour cells to various chemotherapies. Current and future research on the adaptive mechanisms of resistance to PHGDH is needed to harness the upregulation of PHGDH in cancer. A multi-agent metabolic therapy can then be developed utilizing PHGDH as a biomarker for treatment efficacy and potential resistance.

\section{DECLARATIONS}

\section{Acknowledgments}

The authors thank Bethany Prudner and Caitlyn Brashears for helpful discussions.

\section{Authors' contributions}

Conceptualized, wrote, and edited the manuscript: Rathore R, Schutt CR, Van Tine BA

\section{Availability of data and materials}

Not applicable.

\section{Financial support and sponsorship}

The authors would like to thank Dawn Merkel's Bad to the Bone Chili Cook Off, Kellsie’s Hope Foundation, and the National Institute of Health (NIH) - National Cancer Institute (NCI) (R01-CA227115) for funding. 


\section{Conflicts of interest}

Rathore R and Schutt CR declared that there are no conflicts of interest. Van Tine BA declared Basic Science Grant funding from Pfizer, TRACON Pharmaceuticals, and Merck; consulting fees from Epizyme, Eli Lilly, CytRX, Janssen Pharmaceutica, Immune Design, Daiichi Sankyo, Plexxikon, Deciphera Pharmaceuticals, and Adaptimmune; travel support from GlaxoSmithKline; and is an unpaid advisor to Advenchen Laboratories and is on the Scientific Advisory Board of Polaris Inc.

\section{Ethical approval and consent to participate}

Not applicable

\section{Consent for publication}

Not applicable

\section{Copyright}

(c) The Author(s) 2020.

\section{REFERENCES}

1. Hanahan D, Weinberg RA. The hallmarks of cancer. Cell 2000;100:57-70.

2. Pavlova NN, Thompson CB. The emerging hallmarks of cancer metabolism. Cell Metab 2016;23:27-47.

3. Corbin JM, Ruiz-Echevarría MJ. One-carbon metabolism in prostate cancer: the role of androgen signaling. Int J Mol Sci 2016;17:1208.

4. Rosenzweig A, Blenis J, Gomes AP. Beyond the warburg effect: how do cancer cells regulate one-carbon metabolism? Front Cell Dev Biol 2018;6:90.

5. Fan J, Teng X, Liu L, Mattaini KR, Looper RE, et al. Human phosphoglycerate dehydrogenase produces the oncometabolite D-2hydroxyglutarate. ACS Chem Biol 2015;10:510-6.

6. Yang M, Vousden KH. Serine and one-carbon metabolism in cancer. Nat Rev Cancer 2016;16:650-62.

7. Gao X, Lee K, Reid MA, Sanderson SM, Qiu C, et al. Serine availability influences mitochondrial dynamics and function through lipid metabolism. Cell Rep 2018;22:3507-20.

8. Yu L, Teoh ST, Ensink E, Ogrodzinski MP, Yang C, et al. Cysteine catabolism and the serine biosynthesis pathway support pyruvate production during pyruvate kinase knockdown in pancreatic cancer cells. Cancer Metab 2019;7:13.

9. Anderson DD, Stover PJ. SHMT1 and SHMT2 are functionally redundant in nuclear de novo thymidylate biosynthesis. PLoS One 2009;4:e5839.

10. Frezza C. Cancer metabolism: addicted to serine. Nat Chem Biol 2016;12:389-90.

11. Newman AC, Maddocks ODK. Serine and functional metabolites in cancer. Trends Cell Biol 2017;27:645-57.

12. Mazurek S. Pyruvate kinase type M2: a key regulator of the metabolic budget system in tumor cells. Int J Biochem Cell Biol 2011;43:969-80.

13. Chiavarina B, Whitaker-Menezes D, Martinez-Outschoorn UE, Witkiewicz AK, Birbe R, et al. Pyruvate kinase expression (PKM1 and PKM2) in cancer-associated fibroblasts drives stromal nutrient production and tumor growth. Cancer Biol Ther 2011;12:1101-13.

14. Prakasam G, Iqbal MA, Bamezai RNK, Mazurek S. Posttranslational modifications of pyruvate kinase M2: tweaks that benefit cancer. Front Oncol 2018;8:22.

15. Iqbal MA, Gupta V, Gopinath P, Mazurek S, Bamezai RN. Pyruvate kinase M2 and cancer: an updated assessment. FEBS Lett 2014;588:2685-92.

16. Chaneton B, Hillmann P, Zheng L, Martin ACL, Maddocks ODK, et al. Serine is a natural ligand and allosteric activator of pyruvate kinase M2. Nature 2012;491:458-62.

17. Ye J, Mancuso A, Tong X, Ward PS, Fan J, et al. Pyruvate kinase M2 promotes de novo serine synthesis to sustain mTORC1 activity and cell proliferation. Proc Natl Acad Sci U S A 2012;109:6904-9.

18. Sayano T, Kawano Y, Kusada W, Arimoto Y, Esaki K, et al. Adaptive response to l-serine deficiency is mediated by p38 MAPK activation via 1-deoxysphinganine in normal fibroblasts. FEBS Open Bio 2016;6:303-16.

19. Jaeken J, Detheux M, Van Maldergem L, Foulon M, Carchon H, et al. 3-Phosphoglycerate dehydrogenase deficiency: an inborn error of serine biosynthesis. Arch Dis Child 1996;74:542-5.

20. Shaheen R, Rahbeeni Z, Alhashem A, Faqeih E, Zhao Q, et al. Neu-Laxova syndrome, an inborn error of serine metabolism, is caused by mutations in PHGDH. Am J Hum Genet 2014;94:898-904.

21. Acuna-Hidalgo R, Schanze D, Kariminejad A, Nordgren A, Kariminejad MH, et al. Neu-Laxova syndrome is a heterogeneous metabolic disorder caused by defects in enzymes of the L-serine biosynthesis pathway. Am J Hum Genet 2014;95:285-93.

22. Herbig K, Chiang EP, Lee LR, Hills J, Shane B, et al. Cytoplasmic serine hydroxymethyltransferase mediates competition between folatedependent deoxyribonucleotide and S-adenosylmethionine biosyntheses. J Biol Chem 2002;277:38381-9.

23. Sibani S, Melnyk S, Pogribny IP, Wang W, Hiou-Tim F, et al. Studies of methionine cycle intermediates (SAM, SAH), DNA methylation 
and the impact of folate deficiency on tumor numbers in Min mice. Carcinogenesis 2002;23:61-5.

24. Pike MC, Kredich NM, Snyderman R. Requirement of S-adenosyl-L-methionine-mediated methylation for human monocyte chemotaxis. Proc Natl Acad Sci U S A 1978;75:3928-32.

25. Maddocks OD, Labuschagne CF, Adams PD, Vousden KH. Serine metabolism supports the methionine cycle and DNA/RNA methylation through de novo ATP synthesis in cancer cells. Mol Cell 2016;61:210-21.

26. Maddocks OD, Berkers CR, Mason SM, Zheng L, Blyth K, et al. Serine starvation induces stress and p53-dependent metabolic remodelling in cancer cells. Nature 2013;493:542-6.

27. Lowther J, Naismith JH, Dunn TM, Campopiano DJ. Structural, mechanistic and regulatory studies of serine palmitoyltransferase. Biochem Soc Trans 2012;40:547-54

28. Esaki K, Sayano T, Sonoda C, Akagi T, Suzuki T, et al. L-serine deficiency elicits intracellular accumulation of cytotoxic deoxysphingolipids and lipid body formation. J Biol Chem 2015;290:14595-609.

29. Lone MA, Santos T, Alecu I, Silva LC, Hornemann T. 1-Deoxysphingolipids. Biochim Biophys Acta Mol Cell Biol Lipids 2019;1864:512-21.

30. Alecu I, Tedeschi A, Behler N, Wunderling K, Lamberz C, et al. Localization of 1-deoxysphingolipids to mitochondria induces mitochondrial dysfunction. J Lipid Res 2017;58:42-59.

31. Ou Y, Wang SJ, Jiang L, Zheng B, Gu W. p53 Protein-mediated regulation of phosphoglycerate dehydrogenase (PHGDH) is crucial for the apoptotic response upon serine starvation. J Biol Chem 2015;290:457-66.

32. Hanahan D, Weinberg RA. Hallmarks of cancer: the next generation. Cell 2011;144:646-74.

33. Jia XQ, Zhang S, Zhu HJ, Wang W, Zhu JH, et al. Increased expression of PHGDH and prognostic significance in colorectal cancer. Transl Oncol 2016;9:191-6.

34. Xian Y, Zhang S, Wang X, Qin J, Wang W, et al. Phosphoglycerate dehydrogenase is a novel predictor for poor prognosis in gastric cancer. Onco Targets Ther 2016;9:5553-60.

35. Locasale JW, Grassian AR, Melman T, Lyssiotis CA, Mattaini KR, et al. Phosphoglycerate dehydrogenase diverts glycolytic flux and contributes to oncogenesis. Nat Genet 2011;43:869-74.

36. Possemato R, Marks KM, Shaul YD, Pacold ME, Kim D, et al. Functional genomics reveal that the serine synthesis pathway is essential in breast cancer. Nature 2011;476:346-50.

37. Mullarky E, Mattaini KR, Vander Heiden MG, Cantley LC, Locasale JW. PHGDH amplification and altered glucose metabolism in human melanoma. Pigment Cell Melanoma Res 2011;24:1112-5.

38. Issaq SH, Mendoza A, Kidner R, Rosales T, Duveau DY, et al. EWS-FLI1-regulated serine synthesis and exogenous serine are necessary for Ewing sarcoma cellular proliferation and tumor growth. Mol Cancer Ther 2020;19:1520-9.

39. Jing Z, Heng W, Aiping D, Yafei Q, Shulan Z. Expression and clinical significance of phosphoglycerate dehydrogenase and squamous cell carcinoma antigen in cervical cancer. Int J Gynecol Cancer 2013;23:1465-9.

40. Song Z, Feng C, Lu Y, Lin Y, Dong C. PHGDH is an independent prognosis marker and contributes cell proliferation, migration and invasion in human pancreatic cancer. Gene 2018;642:43-50.

41. Sun WY, Kim HM, Jung WH, Koo JS. Expression of serine/glycine metabolism-related proteins is different according to the thyroid cancer subtype. J Transl Med 2016;14:168.

42. Yoon S, Kim JG, Seo AN, Park SY, Kim HJ, et al. Clinical Implication of Serine Metabolism-Associated Enzymes in Colon Cancer. Oncology 2015;89:351-9.

43. Zhang B, Zheng A, Hydbring P, Ambroise G, Ouchida AT, et al. PHGDH defines a metabolic subtype in lung adenocarcinomas with poor prognosis. Cell Rep 2017;19:2289-303.

44. Zhu J, Ma J, Wang X, Ma T, Zhang S, et al. High expression of PHGDH predicts poor prognosis in non-small cell lung cancer. Transl Oncol 2016;9:592-9.

45. Ngo B, Kim E, Osorio-Vasquez V, Doll S, Bustraan S, et al. Limited environmental serine and glycine confer brain metastasis sensitivity to PHGDH inhibition. Cancer Discov 2020;10:1352-73.

46. Nakayama M, Oshima M. Mutant p53 in colon cancer. J Mol Cell Biol 2019;11:267-76.

47. Busuttil RA, Zapparoli GV, Haupt S, Fennell C, Wong SQ, et al. Role of p53 in the progression of gastric cancer. Oncotarget 2014;5:12016-26.

48. Stretch JR, Gatter KC, Ralfkiaer E, Lane DP, Harris AL. Expression of mutant p53 in melanoma. Cancer Res 1991;51:5976-9.

49. Bodner SM, Minna JD, Jensen SM, D'Amico D, Carbone D, et al. Expression of mutant p53 proteins in lung cancer correlates with the class of p53 gene mutation. Oncogene 1992;7:743-9.

50. Dando I, Cordani M, Donadelli M. Mutant p53 and mTOR/PKM2 regulation in cancer cells. IUBMB Life 2016;68:722-6.

51. Wu S, Le H. Dual roles of PKM2 in cancer metabolism. Acta Biochim Biophys Sin 2013;45:27-35.

52. Singh M, Warita K, Warita T, Faeder JR, Lee REC, et al. Shift from stochastic to spatially-ordered expression of serine-glycine synthesis enzymes in 3D microtumors. Sci Rep 2018;8:9388.

53. Mattaini KR, Sullivan MR, Vander Heiden MG. The importance of serine metabolism in cancer. J Cell Biol 2016;214:249-57.

54. Amelio I, Cutruzzolá F, Antonov A, Agostini M, Melino G. Serine and glycine metabolism in cancer. Trends Biochem Sci 2014;39:191-8.

55. Estrela JM, Ortega A, Obrador E. Glutathione in cancer biology and therapy. Crit Rev Clin Lab Sci 2006;43:143-81.

56. Liu S, He L, Yao K. The antioxidative function of alpha-ketoglutarate and its applications. Biomed Res Int 2018;2018:3408467.

57. Badur MG, Muthusamy T, Parker SJ, Ma S, McBrayer SK, et al. Oncogenic R132 IDH1 mutations limit NADPH for De Novo Lipogenesis through (D)2-hydroxyglutarate production in fibrosarcoma sells. Cell Rep 2018;25:1018-1026.e4. 
58. Samanta D, Semenza GL. Serine synthesis helps hypoxic cancer stem cells regulate redox. Cancer Res 2016;76:6458-62.

59. Murphy JP, Giacomantonio MA, Paulo JA, Everley RA, Kennedy BE, et al. The NAD+ salvage pathway supports PHGDH-driven serine biosynthesis. Cell Rep 2018;24:2381-91.e5.

60. Yang L, Garcia Canaveras JC, Chen Z, Wang L, Liang L, et al. Serine catabolism feeds NADH when respiration is impaired. Cell Metab 2020;31:809-21.e6.

61. Samanta D, Park Y, Andrabi SA, Shelton LM, Gilkes DM, et al. PHGDH expression is required for mitochondrial redox homeostasis, breast cancer stem cell maintenance, and lung metastasis. Cancer Res 2016;76:4430-42.

62. Paul MK, Mukhopadhyay AK. Tyrosine kinase - role and significance in cancer. Int J Med Sci 2004;1:101-15.

63. Schöffski P, Dumez H, Clement P, Hoeben A, Prenen H, et al. Emerging role of tyrosine kinase inhibitors in the treatment of advanced renal cell cancer: a review. Ann Oncol 2006;17:1185-96.

64. Wei L, Lee D, Law CT, Zhang MS, Shen J, et al. Genome-wide CRISPR/Cas9 library screening identified PHGDH as a critical driver for Sorafenib resistance in HCC. Nat Commun 2019;10:4681.

65. Cesi G, Walbrecq G, Zimmer A, Kreis S, Haan C. ROS production induced by BRAF inhibitor treatment rewires metabolic processes affecting cell growth of melanoma cells. Mol Cancer 2017;16:102.

66. Larkin JMG, Eisen T. Renal cell carcinoma and the use of sorafenib. Ther Clin Risk Manag 2006;2:87-98.

67. Desideri E, Cavallo AL, Baccarini M. Alike but different: RAF paralogs and their signaling outputs. Cell 2015;161:967-70.

68. Wieduwilt MJ, Moasser MM. The epidermal growth factor receptor family: biology driving targeted therapeutics. Cell Mol Life Sci 2008;65:1566-84.

69. Yang W, Xia Y, Cao Y, Zheng Y, Bu W, et al. EGFR-induced and PKC $\varepsilon$ monoubiquitylation-dependent NF- $\mathrm{kB}$ activation upregulates PKM2 expression and promotes tumorigenesis. Mol Cell 2012;48:771-84.

70. Yang W, Zheng Y, Xia Y, Ji H, Chen X, et al. ERK1/2-dependent phosphorylation and nuclear translocation of PKM2 promotes the Warburg effect. Nat Cell Biol 2012;14:1295-304.

71. Normanno N, Bianco C, De Luca A, Maiello MR, Salomon DS. Target-based agents against ErbB receptors and their ligands: a novel approach to cancer treatment. Endocr Relat Cancer 2003;10:1-21.

72. Wang Y, Schmid-Bindert G, Zhou C. Erlotinib in the treatment of advanced non-small cell lung cancer: an update for clinicians. Ther Adv Med Oncol 2012;4:19-29.

73. Dong J, Lei H, Liang Q, Tang Y, Zhou Y, et al. Overcoming erlotinib resistance in EGFR mutation-positive lung adenocarcinomas through repression of phosphoglycerate dehydrogenase. Theranostics 2018;8:1808-23.

74. Lee JW, Ko J, Ju C, Eltzschig HK. Hypoxia signaling in human diseases and therapeutic targets. Exp Mol Med 2019;51:1-13.

75. Gordan JD, Bertout JA, Hu CJ, Diehl JA, Simon MC. HIF-2alpha promotes hypoxic cell proliferation by enhancing c-myc transcriptional activity. Cancer Cell 2007;11:335-47.

76. Yoshino H, Nohata N, Miyamoto K, Yonemori M, Sakaguchi T, et al. PHGDH as a key enzyme for serine biosynthesis in HIF2 $\alpha$-targeting therapy for renal cell carcinoma. Cancer Res 2017;77:6321-9.

77. Ye J, Fan J, Venneti S, Wan YW, Pawel BR, et al. Serine catabolism regulates mitochondrial redox control during hypoxia. Cancer Discov 2014;4:1406-17.

78. Zhao E, Ding J, Xia Y, Liu M, Ye B, et al. KDM4C and ATF4 Cooperate in transcriptional control of amino acid metabolism. Cell Rep 2016;14:506-19.

79. Selvarajah B, Azuelos I, Platé M, Guillotin D, Forty EJ, et al. mTORC1 amplifies the ATF4-dependent de novo serine-glycine pathway to supply glycine during TGF- $\beta 1$-induced collagen biosynthesis. Sci Signal 2019;12:eaav3048.

80. Xia Y, Ye B, Ding J, Yu Y, Alptekin A, et al. Metabolic reprogramming by MYCN confers dependence on the serine-glycine-one-carbon biosynthetic pathway. Cancer Res 2019;79:3837-50.

81. Wang J, Jiang J, Chen H, Wang L, Guo H, et al. FDA-approved drug screen identifies proteasome as a synthetic lethal target in MYCdriven neuroblastoma. Oncogene 2019;38:6737-51.

82. Chng WJ, Huang GF, Chung TH, Ng SB, Gonzalez-Paz N, et al. Clinical and biological implications of MYC activation: a common difference between MGUS and newly diagnosed multiple myeloma. Leukemia 2011;25:1026-35.

83. Masaki R. Mechanism of action of bortezomib in multiple myeloma therapy. Int J Myeloma 2016;6:1-6.

84. Rizzieri D, Paul B, Kang Y. Metabolic alterations and the potential for targeting metabolic pathways in the treatment of multiple myeloma. J Cancer Metastasis Treat 2019;5:26.

85. Wu X, Xia J, Zhang J, Zhu Y, Wu Y, et al. Phosphoglycerate dehydrogenase promotes proliferation and bortezomib resistance through increasing reduced glutathione synthesis in multiple myeloma. Br J Haematol 2020;190:52-66.

86. Zaal EA, Wu W, Jansen G, Zweegman S, Cloos J, et al. Bortezomib resistance in multiple myeloma is associated with increased serine synthesis. Cancer Metab 2017;5:7.

87. Dahlman KB, Xia J, Hutchinson K, Ng C, Hucks D, et al. BRAF(L597) mutations in melanoma are associated with sensitivity to MEK inhibitors. Cancer Discov 2012;2:791-7.

88. McCain J. The MAPK (ERK) pathway: investigational combinations for the treatment of BRAF-mutated metastatic melanoma. P T 2013;38.

89. Heppt MV, Siepmann T, Engel J, Schubert-Fritschle G, Eckel R, et al. Prognostic significance of BRAF and NRAS mutations in melanoma: a German study from routine care. BMC Cancer 2017;17:536.

90. Cheng Y, Tian H. Current Development Status of MEK Inhibitors. Molecules 2017;22:1551.

91. Nguyen MQ, Teh JLF, Purwin TJ, Chervoneva I, Davies MA, et al. Targeting PHGDH upregulation reduces glutathione levels and 
resensitizes resistant NRAS-mutant melanoma to MAPK kinase inhibition. J Invest Dermatol 2020;S0022-202X(20)31406-8.

92. Ross KC, Andrews AJ, Marion CD, Yen TJ, Bhattacharjee V. Identification of the serine biosynthesis pathway as a critical component of BRAF inhibitor resistance of melanoma, pancreatic, and non-small cell lung cancer cells. Mol Cancer Ther 2017;16:1596-609.

93. Jing Z, Heng W, Xia L, Ning W, Yafei Q, et al. Downregulation of phosphoglycerate dehydrogenase inhibits proliferation and enhances cisplatin sensitivity in cervical adenocarcinoma cells by regulating Bcl-2 and caspase-3. Cancer Biol Ther 2015;16:541-8.

94. Teng PN, Wang G, Hood BL, Conrads KA, Hamilton CA, et al. Identification of candidate circulating cisplatin-resistant biomarkers from epithelial ovarian carcinoma cell secretomes. Br J Cancer 2014;110:123-32.

95. Lee D, Wong CC. The folate cycle is a new metabolic weakness of cancer. Mol Cell Oncol 2017;4:e1327004.

96. Hagner N, Joerger M. Cancer chemotherapy: targeting folic acid synthesis. Cancer Manag Res 2010;2:293-301.

97. Guo W, Healey JH, Meyers PA, Ladanyi M, Huvos AG, et al. Mechanisms of methotrexate resistance in osteosarcoma. Clin Cancer Res 1999;5:621-7.

98. Rohde JM, Brimacombe KR, Liu L, Pacold ME, Yasgar A, et al. Discovery and optimization of piperazine-1-thiourea-based human phosphoglycerate dehydrogenase inhibitors. Bioorg Med Chem 2018;26:1727-39.

99. Mullarky E, Lucki NC, Beheshti Zavareh R, Anglin JL, Gomes AP, et al. Identification of a small molecule inhibitor of 3-phosphoglycerate dehydrogenase to target serine biosynthesis in cancers. Proc Natl Acad Sci U S A 2016;113:E1585.

100. Weinstabl H, Treu M, Rinnenthal J, Zahn SK, Ettmayer P, et al. Intracellular trapping of the selective phosphoglycerate dehydrogenase (PHGDH) inhibitor BI-4924 disrupts serine biosynthesis. J Med Chem 2019;62:7976-97.

101. Wang Q, Liberti MV, Liu P, Deng X, Liu Y, et al. Rational design of selective allosteric Inhibitors of PHGDH and serine synthesis with anti-tumor activity. Cell Chem Biol 2017;24:55-65.

102. Reid MA, Allen AE, Liu S, Liberti MV, Liu P, et al. Serine synthesis through PHGDH coordinates nucleotide levels by maintaining central carbon metabolism. Nat Commun 2018;9:5442.

103. Pacold ME, Brimacombe KR, Chan SH, Rohde JM, Lewis CA, et al. A PHGDH inhibitor reveals coordination of serine synthesis and one-carbon unit fate. Nat Chem Biol 2016;12:452-8.

104. Ravez S, Spillier Q, Marteau R, Feron O, Frédérick R. Challenges and opportunities in the development of serine synthetic pathway inhibitors for cancer therapy. J Med Chem 2017;60:1227-37.

105. Sharif T, Martell E, Dai C, Ghassemi-Rad MS, Lee K, et al. Phosphoglycerate dehydrogenase inhibition induces p-mTOR-independent autophagy and promotes multilineage differentiation in embryonal carcinoma stem-like cells. Cell Death Dis 2018;9:990.

106. Sim WC, Lee W, Sim H, Lee KY, Jung SH, et al. Downregulation of PHGDH expression and hepatic serine level contribute to the development of fatty liver disease. Metabolism 2020;102:154000. 